\title{
Does Cash Incentive Effect TB Case Notification by Public Private Mix-General Practitioners Model in Pakistan?
}

\author{
Robina Ashraf ${ }^{*}$, Farah Naureen², Arif Noor ${ }^{2}$, Jafer Ilyas², Razia Fatima3 ${ }^{3}$, Aashifa Yaqoob3, \\ Ahmad Wali', Mahboob Ul Haq ${ }^{3}$, Abdullah Latif ${ }^{3}$, Sumaira Hussain ${ }^{2}$
}

${ }^{1}$ Islamabad Medical and Dental College, Islamabad, Pakistan

${ }^{2}$ Mercy Corps, Islamabad, Pakistan

${ }^{3}$ National Tuberculosis Control Program Pakistan, Islamabad, Pakistan

${ }^{4}$ Provincial TB Control Program, Balochistan Quetta, Pakistan

Email: *drrobinaashraf@gmail.com

How to cite this paper: Ashraf, R., Naureen, F., Noor, A., Ilyas, J., Fatima, R., Yaqoob, A., Wali, A., Haq, M.U., Latif, A. and Hussain, S. (2018) Does Cash Incentive Effect TB Case Notification by Public Private Mix-General Practitioners Model in Pakistan? Journal of Tuberculosis Research, 6, 166-174.

https://doi.org/10.4236/jtr.2018.62016

Received: March 28, 2018

Accepted: June 19, 2018

Published: June 22, 2018

Copyright (c) 2018 by authors and Scientific Research Publishing Inc. This work is licensed under the Creative Commons Attribution-NonCommercial International License (CC BY-NC 4.0).

http://creativecommons.org/licenses/by-nc/4.0/

\begin{abstract}
Setting: Private healthcare providers including solo practitioners and laboratories enrolled with Public Private Mix General Practitioners model in Pakistan who were enrolled with the program and maintained participation during 2014 to 2016. Objective: To assess the change in case notification by private healthcare providers as well as effect on sputum smear positivity rate at private laboratories after introduction of incentives. Design: A comparative cross-sectional study based on routinely maintained National TB Control Program data collected before and after the introduction of incentives (2014 and 2016). Results: In 2016, TB case notification increased by $71 \%$ after cash incentives was introduced. Among the 618 private healthcare providers who enrolled and maintained participation in the Public Private Mix program, only $1 \%$ of the GPs contributed to $19 \%$ increase in case notification, whereas $27 \%$ GPs had reported a reduced number of cases compared to the pre-intervention period. After incentives, slide positivity rate for diagnostic declined by $1.9 \%$ and follow-up increased by $0.6 \%$. Number of slides for diagnostic and follow-up testing increased by $82 \%$ and $72 \%$ respectively. Conclusion: Engaging the private healthcare sector, by providing cash incentives to private health care Provider's is an effective strategy for providing a substantial increase in National TB case notification.
\end{abstract}

\section{Keywords}

Public Private Mix, Cash Incentive, Tuberculosis, General Practitioner, Pakistan 


\section{Introduction}

Pakistan ranks fifth amongst TB high-burden countries worldwide. In 2015, National TB control (NTP) Pakistan notified 331,809 TB patients, whereas around $1,78,191$ of the incident cases were still missing [1]. Case notification in Pakistan has stagnated over recent years, with the key challenges in TB control being limited engagement of the private healthcare sector, lack of community involvement and escalating number of multidrug-resistant tuberculosis cases [2].

World Health Organization (WHO) recognizes the vital role of engaging the private sector in ending the global TB epidemic and has devised the Public-Private Mix (PPM) approach that aims to engage the private healthcare sector in TB control [3]. In Pakistan, the private sector is the first point of care for $75 \%$ of the population [4]. But $27 \%$ of TB patients being treated in the private sector are not notified to National TB surveillance system [5]. Pakistan implemented the PPM intervention in 2005 and incentives were introduced in 2015, the contribution of different models of PPM to total notified tuberculosis cases was $22 \%$ in 2015 [1] [6].

Engaging private sector participation in notification of $\mathrm{TB}$ cases remains a challenge. As a result, NTP adopted the provision of cash incentives to solo private general physicians (GP) through NTP's partners from July 2015 onwards [7]. However, this strategy has not been evaluated in Pakistan TB control program. The current study aims to assess the effect of cash incentives on tuberculosis case notification by private healthcare provides (PHCP) and sputum slide positivity rate (SPR) at private laboratories enrolled with the PPM-GP model in Pakistan.

\section{Methods}

\subsection{Setting}

Mercy Corps (MC), a private sector partner of NTP and principal recipient of Global Fund grant, is implementing PPM-GP model in 75 districts of Pakistan from all four provinces and three regions through its seven private partners in collaboration with the NTP and Provincial TB Control Program (PTPs).

\subsection{Study Design}

This is a comparative cross-sectional study based on data routinely maintained by Pakistan TB control program. Data was collected for the year before and after cash incentives were introduced (2014 and 2016). In 2015, there was partial implementation of incentives across the country therefore this time period was excluded from the study period.

\subsection{Study Population}

The study population comprises of all PHCP enrolled in PPM-GP model of Mercy Corps from January 01, 2014 and had not dropped out by the end of December 31, 2016. Any PHCPs who joined during the study period or dropped 
out before the end of the study period was excluded from the studied sample. PHCP constitute of GP clinics and laboratories that diagnose, treat and report TB patients. PHCPs (GPs, paramedics, laboratory technicians) are provided training on TB patient management as per the NTP PPM guidelines. The laboratory technicians are provided USD 0.5 incentive per sputum smear slide prepared during diagnosis and follow up evaluation. Incentives to GPs are given in two parts: USD 1 at case registration and USD 3 at declaring treatment outcome to ensure treatment completion. In addition, GPs are provided Anti-TB Drugs (ATT) while laboratories are given consumables and non-consumables free-of-cost. Recording and reporting ( $\mathrm{R} \& \mathrm{R}$ ) tools are provided to PHCP for data collection and extensive monitoring is conducted by NTP, PTPs and Mercy Corps to ensure quality of services provided by PHCP and laboratories. All engaged private laboratories are covered under External Quality Assurance (EQA) through district staff of the public sector.

\subsection{Analysis}

Compiled data was extracted from Recording and Reporting tools (TB-03, TB-04, and TB-07). This data is validated by supervisors from both NTP and MC during monitoring visits of PHCPs and quarter-year data validation meetings. Data was imported into EpiData Anlaysis (version 2.2.2. 187, EpiData Association, Odense, Denmark) and descriptive statistics was used to analyse the data.

\subsection{Ethics Approval}

Ethical approval to use routine PPM-GP model data was obtained from NTP, Pakistan.

\section{Results}

An assessment of the overall tuberculosis case notification by PPM-GP model from 2013 to 2016 shows that twice as many cases were notified to the NTP in 2016 compared to (Figure 1).

During 2014, a total of 618 private healthcare providers (PHCP) were enrolled in the incentive scheme and maintained participation in the PPM program for the duration of January 2014 to December 2016. Among them, more than 50\% (393) were situated in Punjab (Table 1).

An After the introduction of incentives, TB case notification increased by 71\%, from 8168 patients in 2014 to 13,941 patients in 2016 (Table 2). A significant increase in TB case notification was found in Punjab, whereas a decrease was observed in Azad Jammu Kashmir (AJK), corresponding to the number of GPs situated in the provinces. Among the type of PHCP, pulmonologists notified double the number of TB cases as compared to 2014. The TB case contribution remained similar for medical specialist, pediatrician and others. $67 \%$ of PHCP had an increase in case notification, $6 \%$ no change and $27 \%$ reported a 


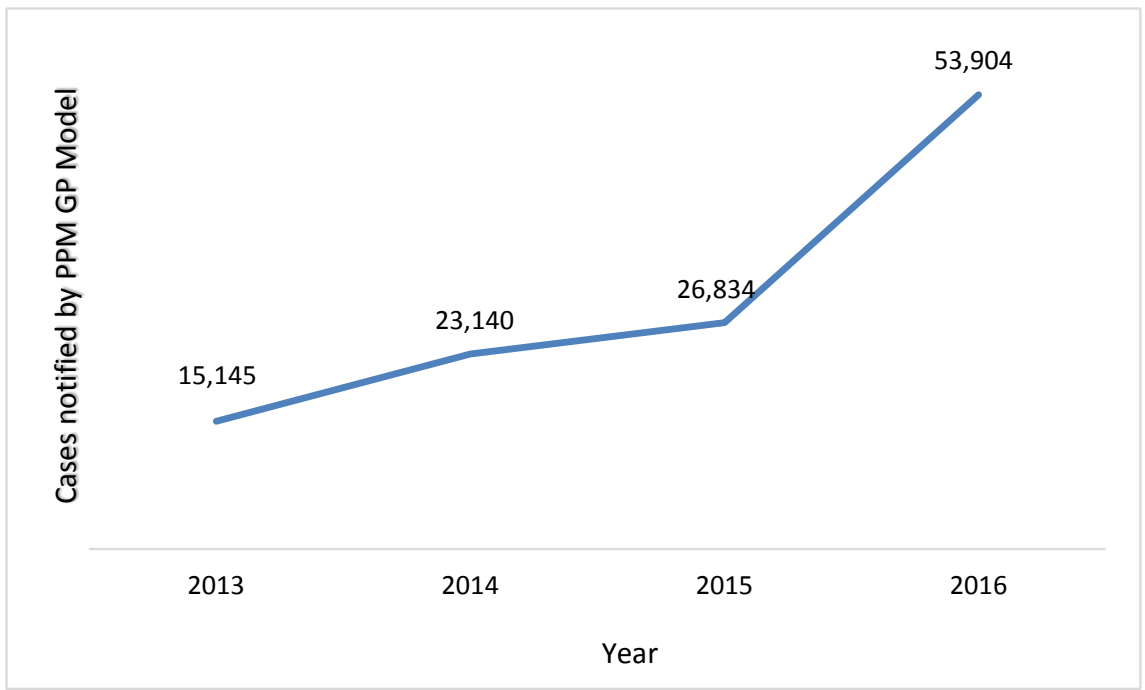

Figure 1. Trend of Tuberculosis case notification by Public Private Mix-General Practitioners model (PPM-GP) to National Tuberculosis Control Program Pakistan (NTP) for 2013-16.

Table 1. Type of private healthcare providers enrolled in Public-Private Mix General Practitioner model in Pakistan, in 2014 and 2016.

\begin{tabular}{ccccccc}
\hline \multicolumn{7}{c}{ Type of HCP* } \\
& GP & Pulmonologist & $\begin{array}{c}\text { Medical } \\
\text { specialist }\end{array}$ & Pediatrician & Others & Total \\
\hline Province \& Regions & & $\mathbf{n}$ & & & \\
AJK & 2 & 0 & 0 & 1 & 1 & 4 \\
Balochistan & 22 & 6 & 4 & 3 & 3 & 38 \\
Gilgit-Baltistan & 6 & 0 & 1 & 0 & 1 & 8 \\
ICT & 6 & 0 & 0 & 0 & 0 & 6 \\
KPK & 13 & 4 & 13 & 3 & 4 & 37 \\
Punjab & 339 & 12 & 12 & 14 & 16 & 393 \\
Sindh & 128 & 3 & 0 & 0 & 1 & 132 \\
Total & 516 & 25 & 30 & 21 & 26 & 618 \\
\hline
\end{tabular}

PHCP = Private healthcare provider; GP = General Practitioner; Others: Surgeon, Orthopedic, Gynecologist, Dermatologis; $\mathrm{t}$ AJK = Azad Jammu Kashmir; ICT = Islamabad Capital Territory.

reduced number of cases. Furthermore, only $1 \%$ of the PHCPs contributed to $19 \%$ increase in case notification (Table 3).

After incentives, diagnostic slide positivity rate declined by $1.9 \%$ and follow-up slide positivity rate increased by $0.6 \%$. Number of slides for diagnostic and follow-up testing increased by $82 \%$ and $72 \%$, respectively (Table 4 ).

\section{Discussion}

The study shows that cash incentives have significantly increased (71\%) 
Table 2. Change in TB case notification by province and type of private healthcare provider enrolled in Public-Private Mix General Practitioner model after introduction of incentives in Pakistan, in 2014 and 2016.

\begin{tabular}{|c|c|c|c|}
\hline & 2014 & 2016 & Change in numbers (\%) \\
\hline & \multicolumn{2}{|c|}{$\mathrm{n} n$} & n (\%) \\
\hline Total notification & 8168 & 13,941 & 5773 \\
\hline \multicolumn{4}{|l|}{ Province } \\
\hline $\mathrm{AJK}$ & 22 & 10 & -12 \\
\hline Balochistan & 180 & 311 & 131 \\
\hline Gilgit-Baltistan & 14 & 46 & 32 \\
\hline ICT & 15 & 76 & 61 \\
\hline KPK & 801 & 1,038 & 237 \\
\hline Punjab & 5698 & 10,010 & 4312 \\
\hline Sindh & 1438 & 2450 & 1012 \\
\hline \multicolumn{4}{|l|}{ Type of HCP } \\
\hline General Practitioner & $6349(77.7)$ & $11,053(79)$ & $4704(74)$ \\
\hline Pulmonologist & $900(11)$ & $1886(14)$ & $986(110)$ \\
\hline Medical Specialist & $471(5.8)$ & $439(3)$ & $-32(-7)$ \\
\hline Pediatrician & $208(2.5)$ & $261(2)$ & $53(25)$ \\
\hline Others & $240(2.9)$ & $302(2)$ & $62(26)$ \\
\hline
\end{tabular}

$\mathrm{AJK}=$ Azad Jammu Kashmir ICT $=$ Islamabad Capital Territory KPK $=$ Khyber Pakhtun Khwa; HCP = Healthcare provider.

Table 3. Change in number of cases notified by healthcare provider enrolled with Mercy Corps Pakistan, before and after introduction of incentives (2014 \& 2016).

\begin{tabular}{|c|c|c|}
\hline & HCP case contribution & Change contribution \\
\hline & \multicolumn{2}{|c|}{ n (\%) n (\%) } \\
\hline $\begin{array}{r}\text { Change in case notific } \\
\text { incentives }\end{array}$ & & \\
\hline Declined & $167(27 \%)$ & $-1248(-22)$ \\
\hline No change & $40(6 \%)$ & $0(0)$ \\
\hline \multicolumn{3}{|c|}{ Increase in cases } \\
\hline $1-10$ & $240(39 \%)$ & $1040(18)$ \\
\hline $11-50$ & $147(24 \%)$ & $3114(54)$ \\
\hline $51-100$ & $15(2 \%)$ & $1114(19)$ \\
\hline $101-200$ & $5(1 \%)$ & $628(11)$ \\
\hline$>200$ & $4(1 \%)$ & $1125(19)$ \\
\hline Total & 618 & 5773 \\
\hline
\end{tabular}

$\mathrm{HCP}=$ Health Care Provider . 
Table 4. Slide positivity rate of private laboratories enrolled in the Public Private Mix-General Practitioner model, before and after introduction of incentives in Pakistan (2014 \& 2016).

\begin{tabular}{ccccc}
\hline & 2014 & 2016 & Change from \\
\hline & & $\mathrm{n}$ & & baseline \\
\hline Diagnostic slides & 16,788 & 30,527 & $\mathbf{0 . 8 2}$ \\
Diagnostic SPR & 14.8 & 12.9 & -1.9 \\
Follow up slides & 3,756 & 6,531 & 0.73 \\
Follow-up SPR & 2.0 & 2.6 & 0.6 \\
\hline
\end{tabular}

SPR = Slide Positivity Rate

tuberculosis case notification in Pakistan. Incentives are provided by Mercy Corps to the PHCPs in order to encourage participation of private sector in tuberculosis case notification as well as to ensure that the program rather than the patient pays for the consultation cost of the of the private provider. There have been multiple systematic reviews that have indicated an improved performance of PHCPs upon provision of cash incentives [8] [9] [10] [11]. Incentives in the form of free drugs, laboratory reagents, medical supplies, gift vouchers or cash payments have led to increased case notification and improved treatment outcomes [7] [12]. Studies show that little improvement in TB case treatment in different countries was observed when provided with incentives of small amounts [11]. While other studies in India reveal that private providers were willing to participate in $\mathrm{TB}$ control activities without any monetary incentives [13] [14].

As the private sector is largely unregulated, there is a probability of non-adherence to NTP management guidelines. This can lead to delay in diagnosis, increased transmission, and transmission of multi-drug resistant tuberculosis due to poor treatment regimens and lack of compliance [15]. Studies have shown that as a result of engagement of private healthcare sector, there is improved access to TB care with minimization of out of pocket an expense owing to free anti-TB drugs and TB diagnostics [8] [11] [16]. Patients under treatment by specialists other than pulmonologists face a delays in being identified as presumptive TB case [17]. Therefore, one of the principles of the End TB strategy is to engage all care providers [18]. This makes incentives an important element of the PPM-GP model to motivate the for-profit PHCP to participate in TB control and make the program a success.

Among the type of healthcare providers, pulmonologists showed the greatest difference in case notification, with the number doubling after incentives. As pulmonologists are the most relevant specialist in the management of tuberculosis, this group is expected to have a high number of cases present, with incentives encouraging the reporting of their cases [17].

Cash incentives to laboratory technicians for sputum smear microscopy demonstrate that there is remarkable increase in the number of sputum smear 
microscopy tests performed with a decrease of slide positivity rate for diagnostic by 1.9 and an increase for follow-up by 0.6 slide positivity rate. A decrease in diagnostic slide positivity rate may be due to high index of suspicion of healthcare providers leading to increased presumptive patient referral. Conversely, the increase in follow-up rate may be due to keen examination of the slides after multiple supervisory visits.

There were several strengths of the study, including a large sample size of all GPs who maintained participation in the program from all four provinces and three regions of Pakistan. In addition, the use of routinely collected data and the results derived from them reflect programmatic realities. Furthermore, large data base of 2 years was used for analysis. A limitation of this study is that review of national program record was relied upon to derive conclusions. Since the data is routinely collected, the accuracy and completeness cannot be ensured. Furthermore, it was not possible to identify other factors that may also have contributed to increased notification other than incentives. These factors are important to elucidate when provided with data that reveals that only $1 \%$ of the GPs contributed to $19 \%$ increase in case notification, whereas 27\% GPs had reported a reduced number of cases.

\section{Conclusion}

This study shows that incentives have been effective in motivating a majority of the private healthcare providers in increasing TB patient notification. Thereby, serving as an important advocacy tool for involvement of the private healthcare providers in TB patient management after appropriate training and accompanied regular monitoring. Further quantitative and qualitative research is recommended to explore factors associated with higher case notification, apart from financial incentives.

\section{Acknowledgements}

This research was conducted through the Structured Operational Research and Training Initiative (SORT IT), a global partnership led by the Special Programme for Research and Training in Tropical Diseases at the World Health Organization (WHO/TDR). The training model is based on a course developed jointly by the International Union Against Tuberculosis and Lung Disease (The Union, Paris, France) and Médecins Sans Frontières (MSF, Geneva, Switzerland). The specific SORT IT programme that resulted in this publication was implemented by the National Tuberculosis Control Programme of Pakistan, through the support of the Global Fund to Fight AIDS, Tuberculosis and Malaria (The Global Fund, Geneva, Switzerland).

The authors acknowledge the support of Mr. Aamir Khan, Project Officer, Mercy Corps in compilation of data for this study. The authors are very much thankful to Muhammad Tariq, Ms. Hina Najmi and Mr. Waseemullah for their valuable inputs as participants of SORT IT 2017. 


\section{Budget}

The publication fee was covered by the NTP through Global Fund, grant Pakis$\tan$

\section{References}

[1] WHO (2016) Global TB Report 2016. WHO, Geneva.

[2] Fatima, R., Qadeer, E., Enarson, D.A., Hinderaker, S.G., Harris, R. and Yaqoob, A.B.A. (2014) Investigation of Presumptive Tuberculosis Cases by Private Health Providers: Lessons Learnt from a Survey in Pakistan. Public Health Action, 4, 14-18.

[3] Board, E. (2015) Global Strategy and Targets for Tuberculosis Prevention, Care and Control after 2015. WHO, Geneva, 1-23.

[4] Naqvi, S.A., Naseer, M., Kazi, A., Pethani, A., Naeem, I., Zainab, S., et al. (2012) Implementing a Public-Private Mix Model for Tuberculosis Treatment in Urban Pakistan: Lessons and Experiences. International Journal of Tuberculosis and Lung Disease, 16, 817-821.

[5] Fatima, R., Harris, R.J., Enarson, D.A., Hinderaker, S.G., Qadeer, E., Ali, K., et al. (2014) Estimating Tuberculosis Burden and Case Detection in Pakistan. International Journal of Tuberculosis and Lung Disease, 18, 55-60.

[6] Global Fund (2014) Standard Investing for impact against HIV, Tuberculosis or Malaria. Global Fund, Geneva, 1-70.

[7] USAID (2014) Public Private Mix (PPM) Models for the Sustainability of Successful TB Control Initiatives Table of Contents. USAID, Washington DC, 1-29.

[8] Beith, A., Eichler, R. and Weil, D. (2009) Worldwide: Incentives for Tuberculosis Diagnosis and Treatment. Performance Incentives for Global Health: Potential and Pitfalls, 237-256.

[9] Lutge, E.E., Wiysonge, C.S., Knight, S.E., Sinclair, D. and Volmink, J. (2015) Incentives and Enablers to Improve Adherence in Tuberculosis. The Cochrane Database of Systematic Reviews, Cochrane.

[10] Lei, X., Liu, Q., Escobar, E., Philogene, J., Zhu, H., Wang, Y., et al. (2015) Public-Private Mix for Tuberculosis Care and Control: A Systematic Review. International Journal of Infectious Diseases [Internet], 34, 20-32. https://doi.org/10.1016/j.ijid.2015.02.015

[11] Beith, A., Eichler, R. and Weil, D. (2007) Performance-Based Incentives for Health: A Way to Improve Tuberculosis Detection and Treatment Completion?

[12] Ridderhof, J. and Humes, R.B.F. (2011) External Quality Assessment for AFB Smear Microscopy.

[13] Balasubramanian, R., Rajeswari, R., Vijayabhaskara, R.D., Jaggarajamma, K., Gopi, P.G., Chandrasekaran, V., et al. (2006) A Rural Public-Private Partnership Model in Tuberculosis Control in South India. The International Journal of Tuberculosis and Lung Disease: The Official Journal of the International Union against Tuberculosis and Lung Disease, 10, 1380-1385.

[14] Murthy, K.J.R., Frieden, T.R., Yazdani, A. and Hreshikesh, P. (2001) Public-Private Partnership in Tuberculosis Control: Experience in Hyderabad, India. International Journal of Tuberculosis and Lung Disease, 5, 354-359.

[15] WHO (2014) Reaching the "Missing Million" through Scaling up Public-Private Mix for TB Care and Control in "High-Impact" Asia. WHO, 53, 1689-1699. 
[16] Lei, X., Liu, Q., Escobar, E., Philogene, J., Zhu, H., Wang, Y., et al. (2015) Public-Private Mix for Tuberculosis Care and Control: A Systematic Review. International Journal of Infectious Diseases, 34, 20-32.

https://doi.org/10.1016/j.ijid.2015.02.015

[17] Chen, T.-C., Lu, P.-L., Lin, W.-R., Lin, C.-Y., Lin, S.-H., Lin, C.-J., et al. (2010) Diagnosis and Treatment of Pulmonary Tuberculosis in Hospitalized Patients Are Affected by Physician Specialty and Experience. The American Journal of the Medical Sciences, 340, 367-372. https://doi.org/10.1097/MAJ.0b013e3181e92b06

[18] Organization WHO (2015) Implementing the End TB Strategy. WHO, Geneva. 